\title{
Clinical application of limiting laryngeal mask airway cuff pressures utilizing inflating syringe intrinsic recoil
}

David M. Corda ${ }^{1}$, Christopher B. Robards ${ }^{2}$, Mark J. Rice ${ }^{3}$, Timothy E. Morey ${ }^{1}$, Nikolaus Gravenstein ${ }^{1}$, Terrie Vasilopoulos $^{1,4}$, Sorin J. Brull ${ }^{2}$

\author{
${ }^{1}$ Department of Anesthesiology, University of Florida College of Medicine, Gainesville, Florida, USA \\ ${ }^{2}$ Department of Anesthesiology and Perioperative Medicine, Mayo Clinic College of Medicine, Jacksonville, Florida, USA \\ ${ }_{3}^{3}$ Department of Anesthesiology, Vanderbilt University Medical Center, Nashville, Tennessee, USA \\ ${ }^{4}$ Department of Orthopaedics and Sports Medicine Institute, Gainesville, Florida, USA
}

\begin{abstract}
Background. Overinflation of the laryngeal mask airway (LMA) cuff may cause many of the complications associated with the use of the LMA. There is no clinically acceptable (cost effective and practical) method to ensure cuff pressure is maintained below the manufacturer's recommended maximum value of $60 \mathrm{~cm} \mathrm{H}_{2} \mathrm{O}(44 \mathrm{mmHg})$. We studied the use of the intrinsic recoil of the LMA inflating syringe as an effective and practical way to limit cuff pressures at or below the manufacturer's recommended values.

Methods. We enrolled 332 patients into three separate groups: LMAs inserted and inflated per standard practice at the institution with only manual palpation of the pilot balloon; LMA cuff pressures measured by a pressure transducer and reduced to $<60 \mathrm{~cm} \mathrm{H}_{2} \mathrm{O}(44 \mathrm{mmHg})$; and LMA intra-cuff pressure managed by the intrinsic recoil of the syringe.

Results. There were no statistically significant differences between the pressure transducer group and the syringe recoil group for initial cuff pressure or cuff pressure 1 hour after surgery. Both the syringe recoil group and pressure transducer group were less likely than the standard practice group to have sore throat and dysphagia 1 hour after surgery. These differences remained 24 hours after surgery.

Conclusions. Syringe recoil provides an efficient and reproducible method similar to manometry in preventing overinflation of the LMA cuff and decreasing the incidence of postoperative laryngopharyngeal complications.
\end{abstract}

Keywords: laryngeal mask airway, sore throat, postoperative complications

\section{Introduction}

The laryngeal mask airway (LMA) is a common supraglottic device used to maintain the airway in patients undergoing general anesthesia. As with any airway device, use of an LMA may cause various

Address for correspondence:

avid M. Corda

Department of Anesthesiology University of Florida College of Medicine

1600 SW Archer Road, PO Box 100254

Gainesville, FL 32610, USA

E-mail: dcorda@anest.ufl.edu perioperative complications. Minor events, including sore throat, have been reported $17.5-42 \%$ of the time an LMA is used [1-3]. Although rare, serious complications including arytenoid dislocation and even nerve damage may occur as well [4-7]. Overinflation of the LMA cuff contributes to postoperative sore throat and other complications associated with LMA placement $[8,9]$. This overinflated and therefore overly pressurized cuff is thought to cause pressure injury by decreasing the perfusion of the soft tissue surrounding the larynx [10, 11]. Previously, investigators have shown that the intrinsic recoil of an LMA inflating syringe can be effective for limiting LMA cuff pressure in vitro. Rice et al. showed in vitro that "the intracuff pressure of the LMA forced the syringe plunger 
to rebound and, more importantly, that the residual LMA cuff pressure would be closer to, or even within, the recommended 'safe zone' of pressure (i.e., $\leq 44$ $\mathrm{mmHg}$ )" [12]. From those observations, we hypothesized that we could similarly limit LMA cuff pressure in vivo using the same syringe recoil method as previously described. We therefore designed a study to determine the effectiveness of using intrinsic recoil of the inflating syringe to limit LMA cuff pressure and reduce the incidence of postoperative pharyngolaryngeal complications as compared to the gold standard method of direct LMA cuff pressure measurement using a sphygmomanometer or pressure transducer. Because the most common method of LMA placement in clinical practice is merely palpation of the LMA pilot balloon with no pressure measurement, we chose to include a third group of patients to compare outcomes as confirmation that either technique of manometry or syringe recoil provides better outcomes than the current standard practice.

\section{Methods}

The clinical study was approved by the IRB of the Mayo Clinic College of Medicine in Jacksonville, Florida, and the University of Florida College of Medicine in Gainesville, Florida. Prior to enrolling patients, the study was registered on June 28, 2011, with clinicaltrials.gov (NCT01385969).

Between June 2011 and April 2013, 332 patients were enrolled in this two-center study at the Mayo Clinic Florida in Jacksonville and at the University of Florida in Gainesville. Inclusion criteria included American Society of Anesthesiologists physical status I-III patients aged 18-80 who were scheduled to receive general anesthesia via LMA for scheduled, short-duration ( $<2$ hours) elective surgery. Exclusion criteria included recent history (within 1 month) of upper respiratory tract infection and any surgical or anesthetic factors considered contraindications to LMA use (e.g., risk of pulmonary aspiration, known difficult airway anatomy, history of uncontrolled gastric reflux, body mass index $>40 \mathrm{~kg} / \mathrm{m}^{2}$, lateral or prone position). Patients undergoing oral or nasal surgery were also excluded. All patients signed a Health Insurance Portability and Accountability Act (HIPAA) waiver and gave informed written consent for participation in the study prior to surgery.

The total number of study patients was divided between the two institutions. Enrolled patients at each center were then subdivided into three equal groups. The first group (standard practice) had LMA cuff pressures set by standard clinical practice in the thencurrent use at each institution without any guidelines provided by the study authors. This involved mainly palpation of the LMA cuff pilot balloon by hand to determine a reasonable LMA cuff pressure. The remaining patients at each center were randomized between the second (pressure transducer) group and the third (syringe recoil) group. Post-insertion LMA cuff pressures for the pressure transducer group were measured and adjusted by a pressure transducer per manufacturer recommendations. LMA cuff pressures for the syringe recoil group were determined by allowing passive, intrinsic recoil of the inflation syringe.

Anesthetic management of patients was standardized. Preoperative anticholinergic drugs were omitted in all patients to prevent drying of oral secretions that may affect ease of LMA insertion or complication rates. As a premeditation for transport to the operating room, we administered 1-2 mg midazolam intravenously. Upon arrival, standard American Society of Anesthesiologists (ASA) monitors were applied to all patients (electrocardiograph, pulse oximeter, and noninvasive blood pressure monitoring). The disposable electronic pressure transducer (Edwards Lifesciences, Irvine, CA) used to measure LMA cuff pressures was recalibrated prior to each case. All patients underwent preoxygenation followed by intravenous induction of anesthesia. If necessary, mask ventilation was used prior to placement of the LMA. If any form of oral airway device was necessary to facilitate mask ventilation prior to LMA insertion, this was noted to avoid confounding evaluation of oropharyngeal complication rates. Following induction of anesthesia, an LMA was placed when the consultant determined adequate depth of anesthesia had been reached (via relaxation of the jaw and loss of eyelash reflexes).

LMA sizes were standardized based on patient weight and sex as in several previous trials [13]. Women weighing greater than $70 \mathrm{~kg}$ received a size 4 LMA Unique (Teleflex ${ }^{\circledR}$, Morrisville, NC). Women weighing less than $70 \mathrm{~kg}$ received a size 3 LMA Unique. Men weighing less than $90 \mathrm{~kg}$ received a size 4 LMA, while those weighing greater than $90 \mathrm{~kg}$ received a size 5 LMA Unique. Anesthesiologists, nurse anesthetists (CRNAs), and anesthesia residents with more than 1 year of experience using LMAs were eligible to insert the LMA. A water-soluble lubricant was applied to the posterior surface of the LMA prior to insertion per manufacturer instructions [14]. The LMA was inserted in all patients using a technique deemed appropriate by the anesthesia provider, guided by both the manufacturer's recommendation and the anesthesia provider's common practice. Following insertion of the LMA in each of the three groups, all LMAs were inflated with a $20-\mathrm{ml} \mathrm{B}$. Braun ${ }^{\mathrm{TM}}$ syringe (B. Braun Medical Inc., Bethlehem, PA) to achieve an effective airway seal as determined by listening for an audible leak during manual ventilation up to $20 \mathrm{~cm} \mathrm{H}_{2} \mathrm{O}$ airway 
pressure. After a seal was achieved, assessment of ventilation was accomplished by observing thoracoabdominal movement with manual ventilation as well as adequate end-tidal carbon dioxide via capnography. In the event that the LMA was not seated properly or an adequate seal was not achieved, the LMA was repositioned. Any repositioning or removal and replacement of an LMA was recorded. General anesthesia was maintained with sevoflurane. Inhaled nitrous oxide was not used.

After induction of anesthesia, ventilation was initiated manually through the circle system and LMA until spontaneous ventilation resumed. The standard practice group of 109 were treated this way. The remaining patients were then randomized into the remaining two groups.

In the pressure transducer group, following resumption of spontaneous ventilation through the LMA, the LMA cuff was attached to a three-way stopcock connected in parallel to a recalibrated disposable pressure transducer (Edwards Lifesciences, Irvine, CA). The LMA cuff pressure was then recorded. If the initial LMA cuff pressure was above $44 \mathrm{mmHg}$, it was immediately reduced by a syringe connected to the same stopcock to ensure an LMA cuff pressure at or below the maximum of $44 \mathrm{mmHg}$. Then, after measurement and adjustment of the LMA cuff pressure, the stopcock and pressure transducer were removed from the LMA and the operative case continued as per usual clinical routine.

In the syringe recoil group, following resumption of spontaneous ventilation through the LMA, anesthesia providers reattached a $20-\mathrm{ml} \mathrm{B}$. Braun ${ }^{\mathrm{TM}}$ Luer lock syringe (B. Braun Medical Inc., Bethlehem, PA) (attached to a three-way stopcock and disposable pressure transducer) to the LMA cuff and allowed the plunger to equilibrate with the LMA cuff pressure (Fig. 1). The resulting cuff pressure measured by the pressure transducer was then recorded. Then, the syringe and pressure transducer were detached from the LMA, and the operative case continued as per usual clinical routine.

In both groups (pressure transducer and syringe recoil group), if the operative procedure lasted longer than 1 hour, the pressure transducer was reapplied to the LMA inflation port at the 1-hour mark and the pressure was recorded.

At the conclusion of surgery, the LMA was removed when deemed appropriate by the anesthesia team (eye opening and mouth opening to verbal command). Patients did not routinely undergo pharyngeal suctioning or placement of any additional airway devices. Patients proceeded to the postoperative recovery room and were treated according to institutional pain management and recovery procedures.

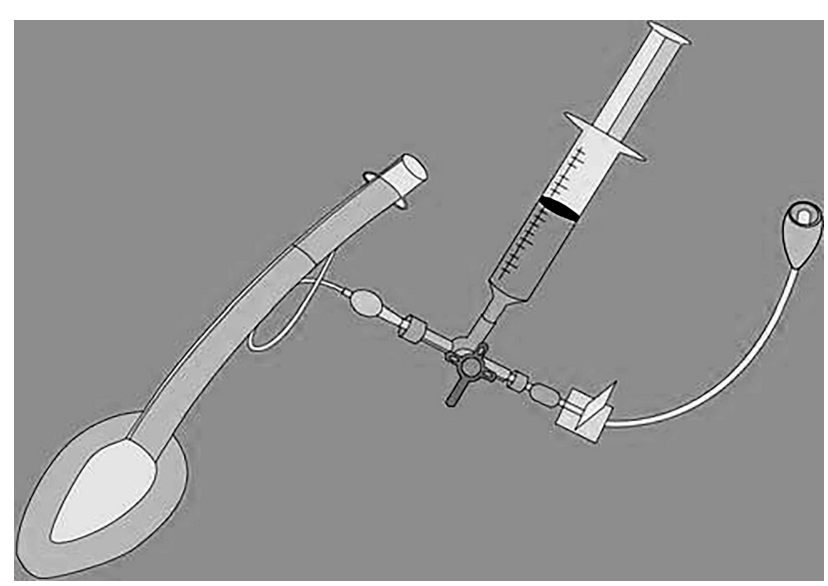

Fig. 1. Transducer hooked up with three-way and inflating syringe in a recoiled position

All outcome data were collected in a de-identified manner. The primary outcome was the LMA cuff pressure in the syringe recoil group after allowing passive recoil of the syringe attached to the LMA cuff. The secondary outcome was the incidence of pharygolaryngeal complications (sore throat, dysphagia, or dysphonia) in all three groups. Sore throat was defined as constant pain or discomfort in the throat independent of swallowing (yes/no). Dysphagia was defined as difficulty in, or pain provoked by, swallowing (yes/no). Dysphonia was defined as difficulty speaking or pain on speaking (yes/no). A blinded research assistant conducted face-to-face interviews with all patients at 1 and 2 hours after surgery and reassessed patients 24 hours after surgery by phone using a predetermined, standardized questionnaire and phone script. The following perioperative data were also collected to rule out possible confounding variables: age of the patient, ASA physical status (PS), gender, height, weight, neck circumference, LMA size used, years of experience of the anesthesia provider placing the LMA, duration of surgery, use of an oral airway device at any time, incidence of laryngospasm, total fentanyl usage, presence or absence of blood on LMA removal, use of pharyngeal suctioning, number of LMA insertion attempts (defined as complete removal and replacement of the LMA), and ease of insertion (rated as easy/fair/difficult).

\section{Statistical analyses}

Analyses were conducted in JMP Pro 13 (SAS Inc., Cary, NC). Continuous measures were summarized by means $\pm \mathrm{SD}$, and categorical measures were summarized as percentages. Differences in perioperative data across groups were analyzed with analysis of variance (ANOVA) or ANOVA on Ranks for continuous measures and Chi-square or Fisher's exact test for categorical variables. Group differences in primary and secondary outcomes were analyzed by either linear 
(continuous outcomes) or logistic (categorical outcomes) regression. The effect size of the relationship between group status and outcomes was assessed by standardized regression coefficients (with standard error) for linear regression and odds ratio with $99 \%$ confidence intervals for logistic regression. $\mathrm{P}<0.05$ was considered statistically significant.

Previous studies determining the incidence of laryngopharyngeal adverse events and LMA cuff pressures were used to determine sample sizes [15]. Initial analyses determined the necessary sample size for our study was 109 patients/group (109 in the standard practice group, 109 in the pressure transducer group, 109 in the syringe recoil group). To account for potential patient attrition, five extra patients were included for a total of 332 patients enrolled in the study. For the primary outcome, a sample size $n=109$ /group would be able to detect an effect size $\mathrm{f}^{2}=0.068$, in a multiple regression model with six covariates, at power $=80 \%$ and alpha $=0.05$. This would translate to a minimally detectable mean difference $=7.5 \mathrm{mmHg}(\mathrm{SD}=15)$ in LMA cuff pressure between the pressure transducer group and the syringe recoil group. However, because initial cuff pressures were not consistently recorded at the Mayo Clinic site, only data from the University of Florida could be used in analysis after study completion ( $n=51$ for pressure transducer group and $n=$ 52 for syringe recoil group). This sample size was able to detect an effect size $\mathrm{f}^{2}=0.068$, in a multiple regression model with six covariates, at power $=80 \%$ and alpha $=0.05$. This would translate to a minimally detectable mean difference $=12 \mathrm{mmHg}(\mathrm{SD}=15)$ in LMA cuff pressure between the pressure transducer group and the syringe recoil group.

\section{Results}

A total of $\mathrm{n}=329$ patients were included in the study, with $\mathrm{n}=180$ from the Mayo Clinic site and $\mathrm{n}=149$ from the University of Florida (three patients were excluded secondary to factors such as cases converting intraoperatively from LMA to endotracheal tube). Table 1 reports perioperative measures across the groups. The standard practice group $(56.1 \pm 17.2$ years $)$ was statistically significantly older than both the pressure transducer group $(49.0 \pm 19.0$ years, $p=0.022)$ and the syringe recoil group $(49.0 \pm 22.2$ years, $p=0.020)$. The standard practice group was less likely to be American Society of Anesthesiologists physical status I $(p=0.004)$ compared to both the pressure transducer and syringe recoil groups. The standard practice group also had higher occurrence of laryngospasm (6.5\%) compared to both the pressure transducer group and syringe recoil group $(p<0.001)$, both of which had no occurrence of laryngospasm. Finally, the standard practice group was also more likely to have a difficult LMA insertion $(8.4 \%, p=0.024)$ compared to the pressure transducer group (1.8\%) and syringe recoil group $(2.7 \%)$. All groups were similar with regards to other perioperative measures.

As mentioned above, only data from the University of Florida could be used in the analysis for the primary outcome of LMA cuff pressure. After adjusting for age, gender, BMI, ASA PS, and ease of insertion, there were no statistically significant differences between the pressure transducer group and syringe recoil group for initial cuff pressure $(53.6 \pm 25.1 \mathrm{mmHg}$ vs. $43.6 \pm$ $20.5 \mathrm{mmHg} ; \beta=2.76 \mathrm{SE}=2.34, \mathrm{p}=0.240$ ) or pressure 1 hour after surgery $(52.3 \pm 23.0 \mathrm{mmHg}$ vs. $42.3 \pm$ $15.6 \mathrm{mmHg} ; \beta=2.76 \mathrm{SE}=2.34, \mathrm{p}=0.240)$. Note: cuff pressure was not measured in the standard practice group.

For secondary outcomes (sore throat, dysphagia, and dysphonia), data from both sites were included. There were overall statistically significant group differences across groups 1 hour after surgery in sore throat $(p=0.004)$ and dysphagia $(p=0.002)$, with a trend towards a difference in dysphonia $(\mathrm{p}=0.06)$ (Fig. 2).

Specifically, compared to the standard practice group, both the pressure transducer and syringe recoil patient groups were less likely to have a sore throat $(\mathrm{OR}=0.38,99 \%$ CI: $0.16-0.93 \& \mathrm{OR}=0.30,99 \% \mathrm{CI}$ : $0.12-0.77$, respectively) and dysphagia $(\mathrm{OR}=0.31$, 99\% CI: 0.11-0.93 \& OR = 0.28, 99\% CI: 0.09-0.85, respectively) 1 hour after surgery. Occurrence of a sore throat $(\mathrm{p}=0.551)$ or dysphagia $(\mathrm{p}=0.801)$ at 1 hour did not statistically significantly differ between pressure transducer and syringe recoil groups. The overall group differences in sore throat remained 24 hours after surgery $(p=0.018)$, specifically with the syringe recoil group less likely to report sore throat compared to the standard practice group $(\mathrm{OR}=0.25$, 99\% CI: 0.07-0.90) (Fig. 3). There were no statistically significant differences between the pressure transducer group and syringe recoil group $(p=0.104)$ nor the standard practice group $(p=0.117)$ for sore throat 24 hours after surgery. There were no overall group differences in dysphagia $(p=0.187)$ and dysphonia $(p$ $=0.304) 24$ hours after surgery.

\section{Discussion}

Dr. Archie Brain (the inventor of the LMA) recommended that users of the LMA limit cuff pressures to less than $60 \mathrm{~cm} \mathrm{H}_{2} \mathrm{O}(44 \mathrm{mmHg})$ to prevent complications [16]. Brain suggested judging inflation pressure by palpation of the LMA pilot balloon. Other authors have suggested using standard inflation volumes to guide LMA cuff inflation. Unfortunately, 
Table 1. Patient characteristics

\begin{tabular}{|c|c|c|c|c|c|c|c|c|c|c|}
\hline & \multicolumn{3}{|c|}{ Standard practice } & \multicolumn{3}{|c|}{ Pressure transducer } & \multicolumn{4}{|c|}{ Syringe recoil } \\
\hline & Mean or $\%$ & SD & $\mathrm{n}$ & Mean or $\%$ & SD & $\mathrm{n}$ & Mean or $\%$ & SD & $\mathrm{n}$ & $\mathrm{p}$ \\
\hline Age (years) & 56.1 & 17.2 & 107 & 49.0 & 19.0 & 110 & 49.0 & 22.2 & 112 & $0.009^{*}$ \\
\hline ASA physical status & & & & & & & & & & 0.002 \\
\hline 1 & $13.1 \%$ & & 14 & $30.9 \%$ & & 34 & $32.4 \%$ & & 36 & \\
\hline 2 & $38.3 \%$ & & 41 & $40.0 \%$ & & 44 & $32.4 \%$ & & 36 & \\
\hline 3 & $47.7 \%$ & & 51 & $29.1 \%$ & & 32 & $35.1 \%$ & & 39 & \\
\hline 4 & $0.9 \%$ & & 1 & $0.0 \%$ & & 0 & $0.0 \%$ & & 0 & \\
\hline Gender & & & & & & & & & & 0.898 \\
\hline Female & $47.7 \%$ & & 51 & $44.5 \%$ & & 49 & $46.4 \%$ & & 52 & \\
\hline Male & $52.3 \%$ & & 56 & $55.5 \%$ & & 61 & $53.6 \%$ & & 60 & \\
\hline BMI & 27.4 & 7.2 & 107 & 27.3 & 5.2 & 109 & 28.2 & 5.6 & 112 & 0.456 \\
\hline Neck circumference $(\mathrm{cm})$ & 37.9 & 4.7 & 107 & 38.1 & 5.0 & 108 & 36.5 & 9.3 & 111 & 0.912 \\
\hline LMA size & & & & & & & & & & 0.961 \\
\hline 3 & $7.5 \%$ & & 8 & $8.2 \%$ & & 9 & $9.9 \%$ & & 11 & \\
\hline 4 & $60.7 \%$ & & 65 & $62.7 \%$ & & 69 & $62.2 \%$ & & 69 & \\
\hline 5 & $31.8 \%$ & & 34 & $29.1 \%$ & & 32 & $28.8 \%$ & & 32 & \\
\hline Provider experience (years) & 6.2 & 6.6 & 103 & 6.0 & 6.3 & 108 & 6.4 & 6.4 & 111 & 0.913 \\
\hline Anesthetic duration (min) & 87.8 & 47.3 & 104 & 88.5 & 44.1 & 108 & 90.2 & 49.1 & 112 & 0.923 \\
\hline Oral airway used & & & & & & & & & & 0.252 \\
\hline No & $93.5 \%$ & & 100 & $94.5 \%$ & & 104 & $90.1 \%$ & & 100 & \\
\hline Yes & $5.6 \%$ & & 6 & $5.5 \%$ & & 6 & $10.8 \%$ & & 12 & \\
\hline Laryngospasm & & & & & & & & & & $<0.001$ \\
\hline No & $93.5 \%$ & & 100 & $100.0 \%$ & & 110 & $100.0 \%$ & & 111 & \\
\hline Yes & $6.5 \%$ & & 7 & $0.0 \%$ & & 0 & $0.0 \%$ & & 0 & \\
\hline Fentanyl use $(\mathrm{mcg})$ & 127.1 & 101.8 & 106 & 102.0 & 84.9 & 110 & 112.5 & 69.3 & 112 & 0.117 \\
\hline Blood on LMA & & & & & & & & & & 0.323 \\
\hline No & $91.6 \%$ & & 98 & $96.4 \%$ & & 106 & $93.7 \%$ & & 104 & \\
\hline Yes & $8.4 \%$ & & 9 & $3.6 \%$ & & 4 & $6.3 \%$ & & 7 & \\
\hline Intraoral suctioning used & & & & & & & & & & 0.587 \\
\hline No & $58.9 \%$ & & 63 & $52.7 \%$ & & 58 & $57.7 \%$ & & 64 & \\
\hline Yes & $40.2 \%$ & & 43 & $47.3 \%$ & & 52 & $42.3 \%$ & & 47 & \\
\hline LMA insertion attempts & & & & & & & & & & 0.326 \\
\hline 1 & $86.9 \%$ & & 93 & $90.9 \%$ & & 100 & $91.0 \%$ & & 101 & \\
\hline 2 & $8.4 \%$ & & 9 & $8.2 \%$ & & 9 & $6.3 \%$ & & 7 & \\
\hline $3+$ & $4.7 \%$ & & 5 & $0.9 \%$ & & 1 & $3.6 \%$ & & 4 & \\
\hline Ease of LMA insertion & & & & & & & & & & 0.024 \\
\hline Easy & $73.8 \%$ & & 79 & $81.8 \%$ & & 90 & $89.2 \%$ & & 99 & \\
\hline Fair & $17.8 \%$ & & 19 & $16.4 \%$ & & 18 & $9.0 \%$ & & 10 & \\
\hline Difficult & $8.4 \%$ & & 9 & $1.8 \%$ & & 2 & $2.7 \%$ & & 3 & \\
\hline
\end{tabular}

Note: P-vales are from ANOVA or ANOVA on Ranks for continuous measures and from Chi-square tests for categorical measures.

* Post hoc comparisons (Tukey HSD test) showed that patients in the standard practice group were older than those in the pressure transducer group $(\mathrm{p}=0.022)$ and the syringe recoil group $(\mathrm{p}=0.020)$

several studies have shown that these techniques fall short in safely limiting intra-cuff pressure $=44 \mathrm{mmHg}$ [17]. Haldar et al. noted during a prospective audit that a significant majority, $77 \%$, of LMA cuff pressures were greater than $60 \mathrm{~cm} \mathrm{H}_{2} \mathrm{O}$ during routine care [18]. In_fact, almost half (48\%) of the intra-cuff pressures were greater than $120 \mathrm{~cm} \mathrm{H}_{2} \mathrm{O}$, which is more than twice the maximum pressure recommended. Lenior et al. confirmed this in a series of 63 patients noted to have an LMA mean cuff pressure of $180 \mathrm{~cm} \mathrm{H}_{2} \mathrm{O}$ at the start of the anesthetic procedure [19]. Thus, LMA cuff pressures commonly present during routine anesthetic care often exceed manufacturers' recom- mendations. Seet and colleagues reported a reduction in postoperative pharyngolaryngeal complications from $46 \%$ with routine LMA cuff care (mean cuff pressure $155 \pm 78 \mathrm{~cm} \mathrm{H}_{2} \mathrm{O}$ pressure) to $13 \%$ when the LMA cuff pressure was limited to $<60 \mathrm{~cm} \mathrm{H}_{2} \mathrm{O}$ pressure. This and other studies used manometry to test and limit LMA cuff pressures. Although effective, manometry is not widely available, and these devices along with others such as pressure transducers are often impractical in the clinical environment. Thus, development of a systematic, cost-effective, and simple method to limit LMA cuff pressure is a worthwhile patient safety goal. 
$50.0 \%$

$45.0 \%$

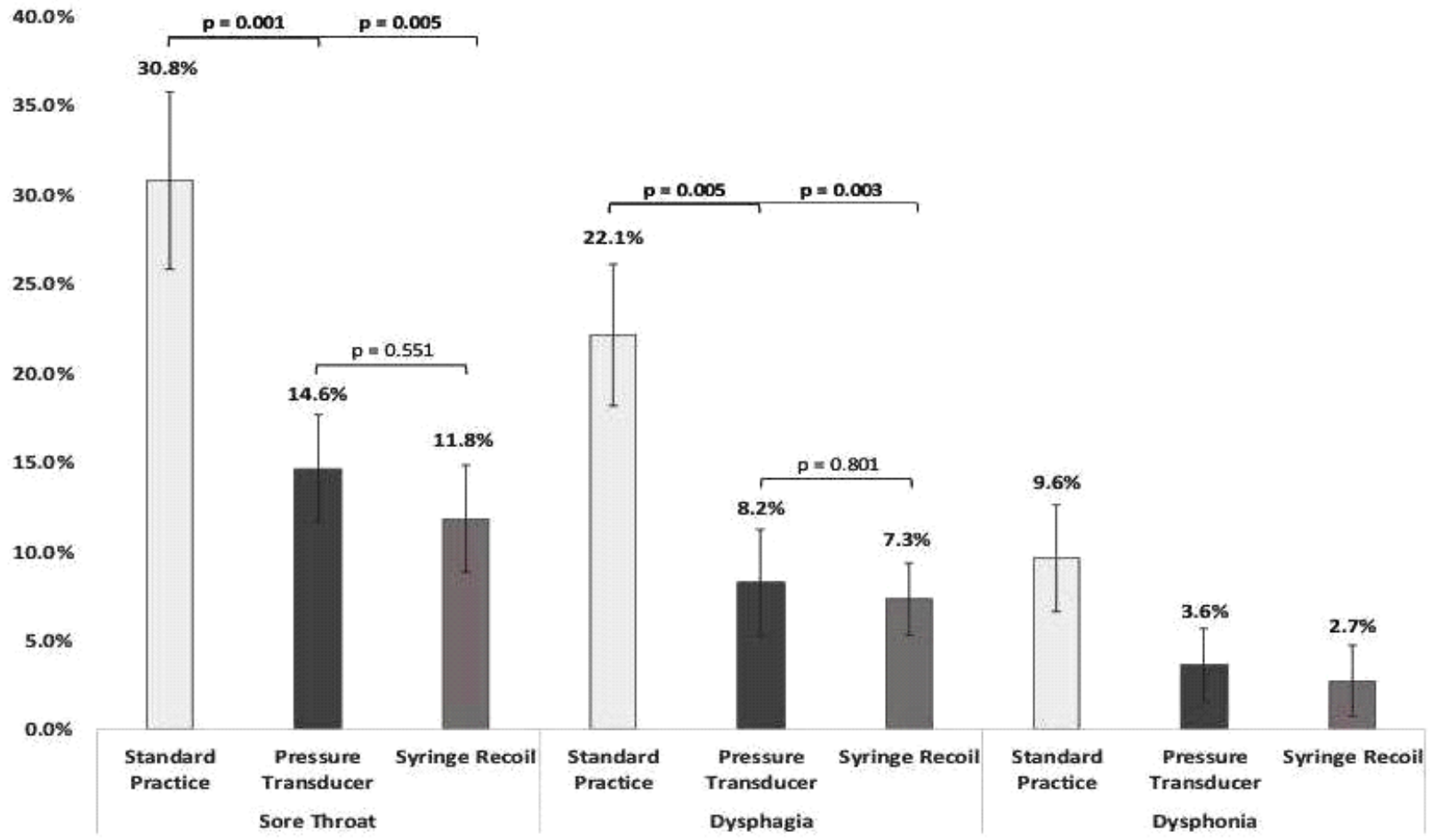

Fig. 2. Percent of patients (\%) with sore throat, dysphagia, and dysphonia 1 hour following surgery (error bars indicate standard error)

$50.0 \%$

$45.0 \%$

$40.0 \%$

$35.0 \%$

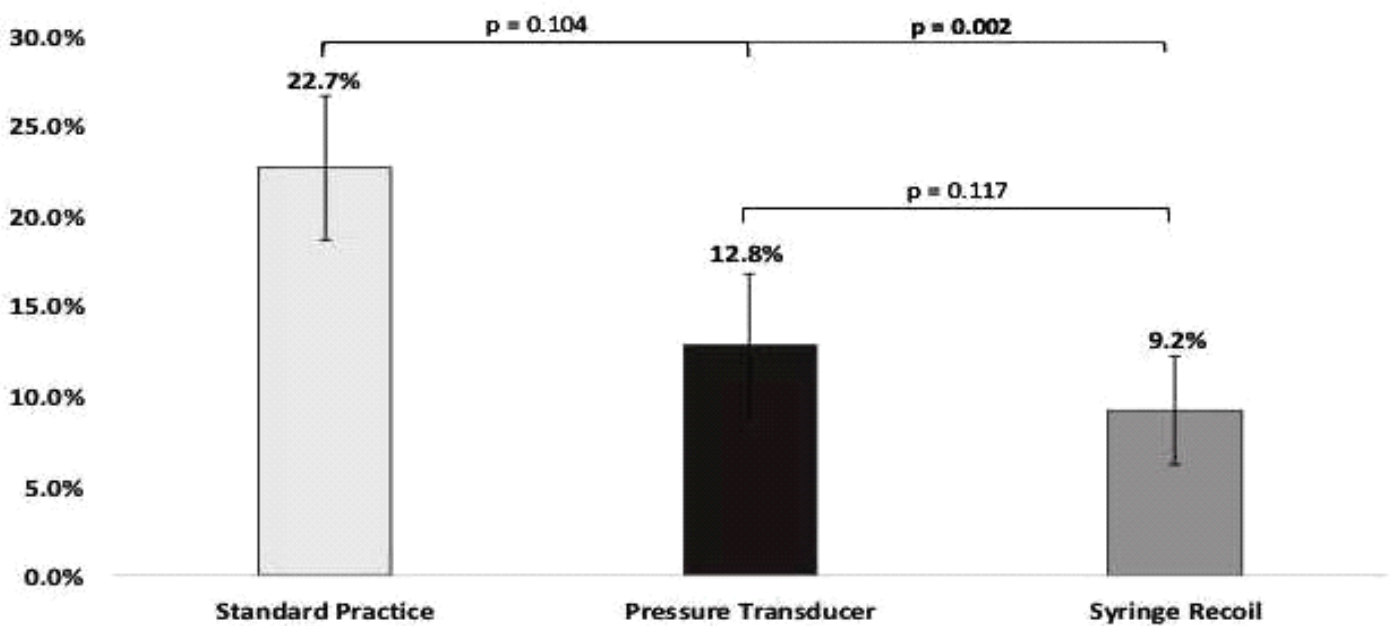

Fig. 3. Percent of patients (\%) with sore throat 24 hours following surgery (error bars indicate standard error) 
While there were omissions in our data collection of cuff pressures at one of the sites, the data collected clearly demonstrate using a syringe recoil method is consistently successful in both reducing the LMA cuff pressure and significantly reducing the occurrence of postoperative complications associated with LMA use as compared to unmonitored cuff pressure. There was no statistically significant difference between the LMA cuff pressure in the pressure transducer group and the group using the syringe recoil method. This is encouraging because it suggests that in practices in which a pressure transducer or manometer may be unavailable, the syringe recoil method may be used to achieve cuff pressures below $44 \mathrm{mmHg}$. However, due to the limited power to detect pressure differences in the present study, future studies with a larger samples size are required to confirm this finding. Furthermore, both the pressure transducer and syringe recoil method groups had a statistically significant lower incidence of postoperative pharyngeal complications as compared to the technique previously used at both institutions, a technique which is likely a clinical standard at most institutions. It is notable that in our demographic data, the standard group was significantly older than the other two groups. Whether or not this affects our data is unknown; however, there are previous studies that have shown increasing age and postoperative pain are negatively correlated [20]. Based on these previous findings, it would be reasonable to hypothesize that our data may actually underestimate the difference in pain experienced between the standard group and the other two groups.

This study was limited by the use of a single LMA, the LMA Unique, along with $20 \mathrm{cc} \mathrm{B}$. Braun inflating syringes. Although previous in vivo studies suggest that various brands and sizes of LMAs and inflating syringes would yield the same results of limiting intracuff pressures to less than $44 \mathrm{mmHg}$, further in vivo studies should be performed to confirm this.

Since conducting this study, clinical practice has changed at both institutions. Namely, a syringe recoil method is routinely recommended during the placement of LMAs. More costly alternatives include providing manometers to each clinical location where LMAs are used or using LMAs with integrated pressure indicators.

In conclusion, our study has confirmed that the suggested syringe intrinsic recoil technique previously shown effectively in vitro not only provides a consistent, efficient, and reproducible method of preventing over inflation of the LMA cuff but also decreases the incidence of postoperative laryngopharyngeal complications after LMA placement when compared to the previous standard practice at both institutions.

\section{Conflict of interest}

Nothing to declare

\section{Funding statement}

This research did not receive any specific grant from funding agencies in the public, commercial, or not-for-profit sectors.

\section{References}

1. Higgins PP, Chung F, Mezei G. Postoperative sore throat after ambulatory surgery. Br J Anaesth 2002; 88: 582-584

2. Grady DM, McHardy F, Wong J, Jin F, Tong D, Chung F. Pharyngolaryngeal morbidity with the laryngeal mask airway in spontaneously breathing patients: does size matter? Anesthesiology 2001; 94: 760-766. doi: 10.1097/00132586200202000-00017

3. Brimacombe J, Holyoake L, Keller C, Brimacombe N, Scully M, Barry J, et al. Pharyngolaryngeal, neck, and jaw discomfort after anesthesia with the face mask and laryngeal mask airway at high and low cuff volumes in males and females. Anesthesiology 2000; 93: 26-31. doi: 10.1097/00000542-200007000-00009

4. Rosenberg MK, Rontal E, Rontal M, Lebenbom-Mansour M. Arytenoid cartilage dislocation caused by a laryngeal mask airway treated with chemical splinting. Anesth Analg 1996; 83: 13351336

5. Rujirojindakul P, Prechawai C, Watanayomnaporn E. Tongue numbness following laryngeal mask airway Supreme ${ }^{\mathrm{TM}}$ and igel ${ }^{\mathrm{TM}}$ insertion: two case reports. Acta Anaesthesiol Scand 2012; 56: 1200-1203. doi: 10.1111/j.1399-6576.2012.02695.x

6. Sommer M, Schuldt M, Runge U, Gielen-Wijffels S, Marcus MA. Bilateral hypoglossal nerve injury following the use of the laryngeal mask without the use of nitrous oxide. Acta Anaesthesiol Scand 2004; 48: 377-378. doi: 10.1111/j.00015172.2004.0332.x

7. Inomata S, Nishikawa T, Suga A, Yamashita S. Transient bilateral vocal cord paralysis after insertion of a laryngeal mask airway. Anesthesiology 1995; 82: 787-788

8. Burgard G, Möllhoff T, Prien T. The effect of laryngeal mask cuff pressure on postoperative sore throat incidence. J Clin Anesth 1996; 8: 198-201. doi: 10.1016/0952-8180(95)00229-4

9. Nott MR, Noble PD, Parmar M. Reducing the incidence of sore throat with the laryngeal mask airway. Eur J Anaesthesiol 1998; 15: 153-157. doi:10.1111/j.0265-0215.1998.00257.x

10. O'Kelly SW, Heath KJ, Lawes EG. A study of laryngeal mask inflation. Pressure exerted on the pharynx. Anesthesia 1993; 48: 1075-1078. doi: 10.1111/j.1365-2044.1993.tb07532.x

11. Brimacombe J, Keller C, Pühringer F. Pharyngeal mucosal pressure and perfusion: A fibreoptic evaluation of the posterior pharynx in anesthetized adult patients with a modified cuffed oropharyngeal airway. Anesthesiology 1999; 91: 1661-1665. doi: 10.1097/00000542-199912000-00018

12. Rice MJ, Gravenstein NL, Brull SJ, Morey TE, Gravenstein N. Using the inflating syringe as a safety valve to limit laryngeal mask pressure. J Clin Monit Comput 2011; 25: 405-410. doi: 10.1007/s10877-011-9319-8

13. Wakeling HG, Butler PJ, Baxter PJ. The laryngeal mask airway: a comparison between two insertion techniques. Anesth Analg 1997; 85: 687-690 
14. Teleflex ${ }^{R}$. Instructions for use - LMA Unique ${ }^{T M}$. Westmeath, Ireland: Teleflex Medical; 2015

15. Seet E, Yousaf F, Gupta S, Subramanyam R, Wong DT, Chung F. Use of manometry for laryngeal mask airway reduces postoperative pharyngolaryngeal adverse events: a prospective, randomized trial. Anesthesiology 2010; 112: 652-657. doi: 10.1097/ALN.0b013e3181 cf4346

16. Brain AIJ. Pressure in laryngeal mask airway cuffs. Anaesthesia 1996; 51: 603. doi: 10.1111/j.1365-2044.1996.tb12581.x

17. Maino P, Dullenkopf A, Keller C, Bernet-Buettiker V, Weiss M. Cuff filling volumes and pressures in pediatric laryngeal mask airways. Paediatr Anaesth 2006; 16: 25-30. doi: 10.1111/j.14609592.2005.01672.x

18. Haldar M, Immanuel P. LMA cuff pressure - An audit (abstract). Anesthesiology 2008; 109: A1720

19. Lenoir RJ. Venous congestion of the neck; its relation to laryngeal mask cuff pressures. Br J Anaesth 2004; 93: 476-477. doi: $10.1093 / \mathrm{bja} / \mathrm{aeh} 603$

20. Ip HY, Abrishami A, Peng PW, Wong J, Chung F. Predictors of postoperative pain and analgesic consumption: a qualitative systematic review. Anesthesiology 2009; 111(3): 657-677. doi: 10.1097/ALN.0b013e3181aae87a 